\title{
Meta-analysis of the efficacy of Xuebijing combined with hemoperfusion in treating paraquat poisoning
}

\author{
Yue Fu ${ }^{1,2,3}$, Mei Yan $^{1,3}$, Xiangxia Zeng ${ }^{1,3}$, Chunming Xie ${ }^{2,3}$, Weigan $\mathrm{Xu}^{2,3}$, Jingxia Feng ${ }^{2,3}$, Jun Jiang ${ }^{2,3}$ \\ ${ }^{1}$ Department of General Medicine, ${ }^{2}$ Department of Emergency, Foshan First People's Hospital, Foshan, China; ${ }^{3}$ The Poison Treatment Centre of \\ Foshan, Foshan, China \\ Contributions: (I) Conception and design: Y Fu, J Jiang; (II) Administrative support: M Yan, X Zeng; (III) Provision of study materials or patients: C \\ Xie, W Xu, J Feng; (IV) Collection and assembly of data: M Yan; (V) Data analysis and interpretation: Y Fu, J Jiang; (VI) Manuscript writing: All \\ authors; (VII) Final approval of manuscript: All authors. \\ Correspondence to: Yue Fu; Jun Jiang. Foshan First People's Hospital, The Poison Treatment Centre of Foshan, No. 81, Lingnan Avenue North, \\ Chancheng District, Foshan 528000, China. Email: drfuy@yahoo.com; jiangjungd@163.com.
}

Background: Paraquat (PQ) is a herbicide that is highly toxic to humans and animals. Xuebijing can regulate immune and inflammatory mediators. Blood purification is a conventional treatment for paraquat poisoning. Therefore, this study aimed to investigate the clinical effect of Xuebijing combined with hemoperfusion on acute paraquat poisoning (APQ).

Methods: The PubMed, Cochrane Library, EMbase, CNKI, CBM, and Wanfang databases were searched by computer for randomized controlled trials (RCT) of Xuebijing combined with hemoperfusion in the treatment of APQ. The search time was from the establishment of database to April 2020. The documents were screened and extracted according to the inclusion and exclusion criteria. Meta-analysis and Revman 5.3 were used to evaluate the quality.

Results: The metanalysis included 10 studies, totaling 636 patients. Results showed that the 7-day survival rate of Xuebijing combined with hemoperfusion group was higher than that of the control group [hazard ratio $(\mathrm{HR})=1.17,95 \%$ confidence interval $(\mathrm{CI})$ : $(1.04,1.32), \mathrm{P}<0.008$ ], while 14 -day survival rate was higher $[\mathrm{HR}=1.52,95 \% \mathrm{CI}:(1.13,2.06), \mathrm{P}<0.006]$, alanine aminotransferase (ALT) was lower [mean difference $(\mathrm{MD})=-32.5,95 \% \mathrm{CI}:(-52.24,-12.76), \mathrm{P}=0.001]$, creatinine was lower $[\mathrm{MD}=-60.73,95 \% \mathrm{CI}:(-103.42$, -18.04), $\mathrm{P}<0.005]$, oxygen partial pressure $\left(\mathrm{PaO}_{2}\right)$ was higher [MD =6.21, 95\% CI: $\left.(1.78,10.64), \mathrm{P}=0.006\right]$, and C-reactive protein $(\mathrm{CRP})$ was lower $[\mathrm{MD}=-6.15,95 \% \mathrm{CI}:(-7.14,-5.16), \mathrm{P}<0.00001]$. However, there was no statistical difference in oxygen saturation $\left(\mathrm{SpO}_{2}\right)$ and carbon dioxide $\mathrm{PaO} 2$ between the two groups.

Conclusions: Xuebijing combined with hemoperfusion and conventional treatment can improve the 7-day and 14-day survival rate, oxygenation level, liver and kidney function, and inflammatory response of paraquat poisoning (PQ) patients.

Keywords: Xuebijing injection; hemoperfusion; paraquat poisoning (PQ); meta-analysis

Submitted May 21, 2020. Accepted for publication Jul 08, 2020.

doi: 10.21037/apm-20-1247

View this article at: http://dx.doi.org/10.21037/apm-20-1247

\section{Introduction}

Paraquat is a highly effective and widely used contact toxic herbicide that has strong toxicity to human and animals. Paraquat poisoning (PQ) can be caused by improper skin contact and inhalation through the respiratory tract throughout the course of life. PQ disease progresses rapidly and depends on the amount of toxin ingested. People can die from oral administration of 5-15 mL PQ, with a mortality rate of over $80 \%$ (1). Paraquat can cause multiple organ dysfunction syndrome (MODS) in a short period of time. The main target organs of damage are the lung, liver, kidney, and heart, with the highest concentration being found in lung 
tissue (2). Generally, toxic compounds accumulate in free radical-forming lung tissues and produce alveolitis, which leads to pulmonary fibrosis (3). Although there are many different treatment methods, the death rate of PQ is still very high, and it remains a serious threat to life (4).

After accidentally being absorbed into blood, PQ mainly accumulates in the lungs. Respiratory failure caused by irreversible advanced pulmonary fibrosis and multiple organ failure mainly caused by acute renal failure are the main causes of PQ-related death. Existing studies have speculated that oxidative stress and inflammatory injury are the main pathogenic mechanisms (5). In brief, PQ enters the body, forms free radicals through single-electron reduction, reacts with molecular oxygen to generate bipyridine cations and superoxide anions. These undergo a disproportionation reaction with superoxide dismutase (SOD) to form hydrogen peroxide, and then produce highly active free radicals in the presence of ferrous ions that initiate chain lipid peroxidation. Finally, damage to the cell membrane system changes the lipid microenvironment of membrane receptors, comprising membrane proteases and ion channels, causing oxidative damage to tissues and cells, especially of the lung (6-8). This in turn leads to pulmonary hemorrhage, edema, hyaline membrane degeneration, or fibrocyte proliferation (9). During this period, a variety of cytokines such as interleukin (IL)-6, IL-10, tumor necrosis factor- $\alpha$ (TNF- $\alpha$ ), IL-1 $\beta$, and insulin-like growth factors (IGF)-1 participate in the development of pulmonary fibrosis after PQ poisoning. Cytokines can interact with corresponding receptors on the surface of target cells through autocrine or paracrine, initiate intracellular signal transduction cascade, and regulate the expression of intracellular factors, thus inducing pulmonary fibrosis (10). In addition, PQ intensively stimulates and corrodes skin and mucosa, which may influence the coagulation mechanism. Heparin is required for anticoagulation during hemoperfusion, which may lead to gastrointestinal hemorrhage, thrombocytopenia, anemia, and other complications (2).

Currently, there is no specific therapeutic drug or therapy for acute PQ poisoning (1). Patients are usually treated aggressively with conventional therapies, including gastric lavage to remove poisons that have not been absorbed by the gastrointestinal tract, intravenous injection of furosemide or furosemide to promote the excretion of $\mathrm{PQ}$, and intragastric infusion of kaolin to promote PQ inactivation. In addition, antioxidants such as quercetin and cyclophosphamide, corticosteroids and other drugs have been administered as treatment (11). Cho et al. found that nitric oxide $(\mathrm{NO})$ inhaled by $\mathrm{PQ}$-poisoned mice could also improve survival rate for 72 hours, reduce malondialdehyde (MDA), and TGF- $\beta 1$ levels in alveolar lavage fluid and blood, and prevent pulmonary fibrosis (12).

Blood purification, including hemoperfusion, hemodialysis, and other measures, is also a routine treatment for PQ poisoning. Hemoperfusion is mainly used for the clearance of medium molecular substances, small molecular circular structure substances, and parts of large molecular substances bound by plasma proteins; hemoperfusion is indicated for PQ, as PQ is considered a medium molecular substances (13). In a short period of time, hemoperfusion can effectively remove endogenous and exogenous poisons in the circulatory system; clear endotoxins, serum bilirubin, and inflammatory cytokines to a degree; form an obstruction effect on the initial link of poisoning; significantly reduce the clinical symptoms of $\mathrm{PQ}$ poisoning; protect against damage from oxidative stress and inflammatory reactions to the lung, liver, kidney, and other important organs and tissues; and can ultimately save lives (14). Although hemoperfusion can reduce the concentration of $\mathrm{PQ}$ in the body, $\mathrm{PQ}$ is widely distributed in the body and has limited clearance capacity (8). The effectiveness of traditional treatment methods is greatly limited, and these interventions have not been proven to improve the survival rate, so further measures to block PQ absorption are needed (15).

In recent years, Xuebijing injection therapy based on antioxidant theory has been used in the clinical treatment of PQ poisoning. Xuebijing injection is a traditional Chinese medicine preparation, based on an ancient prescription called xufu zhuyu tang (decoction for removing blood stasis in the chest), which is composed of red peony root, Chinese angelica, chuanxiong rhizome, safflower, danshen root, and other ingredients. Based on the theory of "simultaneous treatment of bacteria, endotoxins, and inflammatory media", it has the function of regulating immunity and inflammatory media (16). Studies have shown that at least 21 compounds, including amino acids, phenolic acids, flavonoid glycosides, terpenoid glycosides, and phthalates, have been found in Xuebijing (17), and pharmacologic active monomers such as paeoniflorin, ligustrazine ferulic acid, and safflower yellow pigment $\mathrm{A}$, danshensu, and others, can be extracted (18). The main determinants of product quality are safflower yellow pigment A, ligustrazine, danshensu, ferulic acid, and paeoniflorin, most of which have antioxidant properties $(19,20)$. In addition, ferulic acid and ligustrazine have the effect of scavenging oxygen-free radicals, and preventing and treating pulmonary fibrosis and lung injury (21). 
In addition, Xuebijing can reduce the level of endotoxins in the blood; ameliorate sepsis conditions such as fever, leukocyte reaction, and shock caused by endotoxininduced activation of mononuclear giant cells and release of inflammatory transmitters; and improve prognosis (22). Xuebijing can also increase blood flow, reduce the adhesion and aggregation of platelets, inhibit the synthesis of collagen by fibroblasts, decrease the permeability of capillaries during acute inflammation, reduce inflammatory exudation, promote inflammatory absorption, inhibit the formation of inflammatory granuloma, and enhance the phagocytic function and adsorption capacity of reticuloendothelial cells (2).

At present, some studies have shown that the combination of Xuebijing and hemoperfusion therapy is synergistic and can reduce the degree of pulmonary fibrosis, practically eliminate the concentration of $\mathrm{PQ}$ and oxygenfree radicals in the body, reduce the damage to the body, and effectively treat PQ poisoning (8). An efficacy evaluation for the combination of Xuebijing and hemoperfusion therapy on 26 PQ-poisoned patients indicated that this treatment could achieve good results, and the survival rate of the combined treatment group was significantly improved. It has been proven that Xuebijing injection combined with hemoperfusion and other therapies to treat acute PQ poisoning can effectively reduce the functional damage to all organs; it is thus a relatively effective treatment for acute PQ poisoning, and is worth promoting in clinical practice (23). A considerable number studies have observed the curative effect of Xuebijing combined with hemoperfusion on PQ, but no meta-analysis of these published studies has been conducted. Therefore, the purpose of this paper was to comprehensively collect the relevant studies, systematically evaluate and meta-analyze them based on a strict design, and determine the influence of Xuebijing combined with hemoperfusion on the vital organ function and survival of patients with acute PQ poisoning. Through this, we hope to provide a reference for the clinical treatment of PQ using Xuebijing combined with hemoperfusion.

We present the following article in accordance with the PRISMA reporting checklist (available at http://dx.doi. org/10.21037/apm-20-1247).

\section{Methods}

\section{Inclusion and exclusion criteria}

\section{Research type}

The studies included in the meta-analysis were randomized controlled trials (RCTs) of Xuebijing combined with hemoperfusion for the treatment of $\mathrm{PQ}$ poisoning, regardless of blinding or allocation concealment.

\section{Research subjects}

The research subjects had an obvious history of exposure to PQ and showed typical clinical symptoms of PQ poisoning. Combined with laboratory auxiliary examination, the patients diagnosed with PQ were distinguished from those patients with a clear history of heart, lung, liver, kidney, brain, and other basic diseases.

\section{Intervention}

Both the experimental group and the control group were given the same basic routine treatment. The experimental group was treated with Xuebijing injection + hemoperfusion + conventional basic treatment, while the control group was treated with hemoperfusion + conventional basic treatment. The two groups were comparable in age, sex, drug dosage, liver and kidney function, and respiratory and circulatory system function before treatment.

\section{Outcome indexes}

The main outcome indexes included (I) alanine aminotransferase (ALT), (II) creatinine, (III) partial pressure $\left(\mathrm{PaO}_{2}\right)$, (IV) oxygen saturation $\left(\mathrm{SpO}_{2}\right),(\mathrm{V})$ 7-day survival rate, (VI) C-reactive protein (CRP), (VII) 14-day survival rate, and (VIII) partial pressure of carbon dioxide $\left(\mathrm{PaCO}_{2}\right)$.

\section{Exclusion criteria}

The exclusion criteria were the following: (I) literature that republished and used the same test data; (II) literature that only consisted of an abstract without full text; (III) literature with incomplete data or clearly wrong data; (IV) non-RCTs; (V) reviews and conference papers; (VI) animal model experiments and non-original literature; (VII) presence of intervention measures in the experimental group other than Xuebijing injection + hemoperfusion + conventional basic treatment; (VIII) presence of intervention measures in the control group other than hemoperfusion + conventional basic treatment.

\section{Retrieval strategy}

The PubMed, Cochrane Library, EMbase, CNKI, CBM, and Wanfang databases were searched by computer to find RCTs of Xuebijing combined with hemoperfusion in the treatment of Acute Paraquate Poisoning (APQ). The 


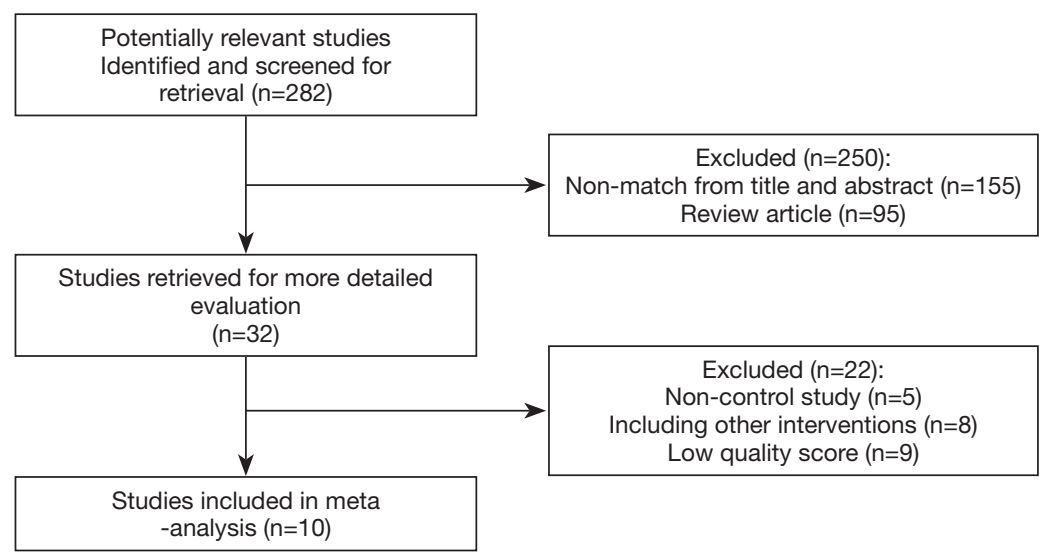

Figure 1 Literature retrieval process.

retrieval period was from the establishment of the database to April 2020. Chinese search words included "Xuebijing", "Xuebijing Injection", "Hemoperfusion”, "Acute Paraquat Poisoning, APQ", "Acute Paraquat Intoxication", "Paraquat Poisoning, PQ", and "Clinical trial, Clinical study"; English search words included "Xuebijing", "Xuebijing Injection", "Hemoperfusion", "Acute Paraquat Poisoning, APQ", "Acute Paraquat Intoxication", "Paraquat Poisoning, PQ", and "Clinical trial, Clinical study". According to the characteristics of different materials, the subject words were combined with free words to search the literature.

\section{Literature selection and data extraction}

The title and abstract of the articles were read, and relevant documents were excluded according to the exclusion criteria. The full text was then read to determine whether the article should be included. The type of data included in the study were the following: (I) basic information, including author and year of publication; (II) number of patients in each group, age, gender, and dosage of paraquat taken; (III) research methods used; (VI) intervention measures, including administration route of Xuebijing and hemoperfusion, dosage, and course of treatment; (V) data of all outcome indexes before and after treatment. The extraction of the above information was carried out by two researchers independently, and any missing information was sought out by contacting the authors.

\section{Literature quality evaluation}

The Cochrane risk bias assessment tools were used to evaluate the quality of the RCTs (24). The Cochrane criteria include random sequence generation, allocation concealment, blinding, blinding assessment of research outcome, integrity of outcome data, selective reporting of research results, and other sources of bias. All included studies were judged according to each criterion.

\section{Statistical analysis}

Stata 15.0 software was used for meta-analysis, and Revman 5.3 was used for quality evaluation. Hazard ratio (HR), mean difference (MD), 95\% confidence interval (CI), and $\mathrm{I}^{2}$ were used to determine interstudy heterogeneity. If there was no heterogeneity $\left(\mathrm{I}^{2}<50 \%\right)$, fixed effect model (FEM) was used for analysis; otherwise, a random effects model (REM) was used.

\section{Results}

\section{Literature screening results}

A total of 282 relevant studies were obtained, 250 of which were excluded after their titles, abstracts, or full-text were read, and 22 of which were excluded for lacking a control group, having other intervention measures, or being of low quality. Finally, 10 studies were included for meta-analysis. A total of 636 patients were included: 319 patients in the test group and 317 patients in the control group. The literature selection process is shown in Figure 1.

\section{Basic characteristics of the included studies}

The 10 included studies $(23,25-33)$ comprised a total of 
636 patients, with 319 cases in the experimental group and 317 cases in the control group. The intervention measures in the experimental group were Xuebijing injection + hemoperfusion + conventional treatment. The intervention measures of the control group were hemoperfusion + conventional treatment, as shown in Table 1.

\section{Methodological quality evaluation of included studies}

Of the 10 included studies (23,25-33), none explained the randomization methods used. Two studies $(28,29)$ apparently adopted an allocation concealment method, while the other studies $(23,25-27,30-33)$ did not mention whether allocation concealment was adopted. Blinding was performed in all studies; one study (30) mentioned that breaking the blind was unlikely, and the others did not mention further blinding details. There were no missing data in 6 studies $(23,27,28,30-32)$, and the other studies did not mention missing data. The main outcome indexes of one study (26) were incompletely reported, and the main outcome indexes of the other studies were fully reported. There was not enough information to determine whether any other biases existed (Figure 2).

\section{Results of meta-analysis}

\section{Survival rate}

Of the 10 included (23,25-33) studies, 5 studies $(25,28,29,31,32)$ reported a 7 -day survival rate, and 5 studies (28-32) reported a 14-day survival rate. The results showed that the 7-day survival rate of Xuebijing combined with hemoperfusion was higher than that of the control group [HR $=1.17,95 \% \mathrm{CI}$ : $(1.04,1.32), \mathrm{P}<0.008]$, and the 14-day survival rate of Xuebijing combined hemoperfusion was higher than that of the control group $[\mathrm{HR}=1.52,95 \%$ CI: $(1.13,2.06), \mathrm{P}<0.006]$. This indicates that Xuebijing combined with hemoperfusion can improve the survival rates of APQ patients at 7 and 14 days (Figure $3 A, B$ ).

\section{Liver and kidney functions}

Of the 10 included studies (23,25-33), 5 studies $(23,25,27,28,30)$ reported ALT and creatinine values. The heterogeneity among the studies was high, and the REM was used for the combined analysis. The results showed that the total ALT level of the Xuebijing combined with hemoperfusion group was lower $[\mathrm{MD}=-32.5,95 \% \mathrm{CI}$ : $(-52.24,-12.76), \mathrm{P}=0.001]$. The total creatinine of the Xuebijing combined hemoperfusion group was lower [MD
$=-60.73,95 \%$ CI: $(-103.42,-18.04), \mathrm{P}<0.005]$, suggesting that Xuebijing combined hemoperfusion could protect liver and kidney functions to a certain extent (Figure 3C,D).

\section{Oxygenation level}

Of the 10 included studies $(23,25-33) 4$ studies $(25,27,30,33)$ reported $\mathrm{PaO}_{2}$, and 2 studies $(28,30)$ reported $\mathrm{SpO}_{2}$. There was heterogeneity among the studies, and the REM was used for the combined analysis. The results showed that the $\mathrm{PaO}_{2}$ value of the combined hemoperfusion group was higher than that of the control group $[\mathrm{MD}=6.21,95 \% \mathrm{CI}$ : $(1.78,10.64), \mathrm{P}=0.006]$. However, there was no significant difference in $\mathrm{SpO}_{2}$ value between the two groups [MD $=4.93,95 \%$ CI: $(-4.59,14.45), \mathrm{P}=0.31]$. The results showed that the combination of hemoperfusion and Xuebijing could improve the oxygen carrying capacity and oxygenation of the body to some extent (Figure 4A,B).

\section{Inflammatory response}

Of the 10 included studies (23,25-33), 2 studies $(28,30)$ reported CRP. There was no obvious heterogeneity among the studies, and the FEM was used for the combined analysis. The results showed that the total CRP of the Xuebijing combined with hemoperfusion group was lower than that of the control group [MD $=-6.15,95 \% \mathrm{CI}:(-7.14$, -5.16), $\mathrm{P}<0.00001]$. This indicates that Xuebijing combined with hemoperfusion can reduce the inflammatory response of the body (Figure 4C).

\section{$\mathrm{PaCO}_{2}$ level}

Of the 10 included studies (23,25-33), 4 studies $(23,27,30,33)$ reported $\mathrm{PaCO}_{2}$, and there was heterogeneity among the studies. The REM was used for the combined analysis. The results showed that there was no statistically significant difference in $\mathrm{PaCO}_{2}$ value between the Xuebijing combined hemoperfusion group and the control group [MD $=-1.112,95 \%$ CI: $(-2.80,0.58), \mathrm{P}=0.197]$, suggesting that the effect of Xuebijing combined with hemoperfusion on improving carbon dioxide accumulation in the body was not obvious (Figure 4D).

\section{Sensitivity analysis}

In the meta-analysis of ALT, creatinine $\mathrm{PaO}_{2}, \mathrm{SpO}_{2}$, and $\mathrm{PaCO}_{2}$ with high heterogeneity, the meta-analysis was carried out again after one study was removed in turn, and the overall heterogeneity did not change significantly. Therefore, the meta-analysis results of various outcome 
Table 1 Basic characteristics of the included literature

\begin{tabular}{|c|c|c|c|c|c|c|c|c|}
\hline First author & Year & $\mathrm{N}$ & Male/female & Age & $\begin{array}{c}\text { Paraquat dose } \\
\text { (observation/control) } \mathrm{mL}\end{array}$ & Intervention measures & $\begin{array}{l}\text { Course of } \\
\text { treatment/d }\end{array}$ & Outcome \\
\hline Li XL & 2008 & 30 & $7 / 23$ & $17-45$ & $30-120$ & $\begin{array}{l}\text { Xuebijing + hemoperfusion } \\
\text { + routine treatment, bid; } \\
\text { hemoperfusion + routine } \\
\text { treatment, bid }\end{array}$ & $7-10$ & $1,2,3,5,8$ \\
\hline Jin LT & 2012 & 70 & $32 / 38$ & $20-54$ & $20-100$ & $\begin{array}{l}\text { Xuebijing + hemoperfusion } \\
\text { + routine treatment, bid; } \\
\text { hemoperfusion + routine } \\
\text { treatment, bid }\end{array}$ & $7-10$ & $1,2,3,8$ \\
\hline Liu ZY & 2015 & 102 & $37 / 65$ & $15-62$ & $20-40$ & $\begin{array}{l}\text { Xuebijing + hemoperfusion } \\
\text { + routine treatment, bid; } \\
\text { hemoperfusion + routine } \\
\text { treatment, bid }\end{array}$ & 7 & 5,7 \\
\hline Lin FC & 2016 & 52 & $21 / 31$ & $23-40$ & $30-150$ & $\begin{array}{l}\text { Xuebijing + hemoperfusion } \\
\text { + routine treatment, bid; } \\
\text { hemoperfusion + routine } \\
\text { treatment, bid }\end{array}$ & 7 & $1,2,3,8$ \\
\hline Shi WL & 2018 & 68 & $33 / 35$ & $18-75$ & $9-37$ & $\begin{array}{l}\text { Xuebijing + hemoperfusion } \\
\text { + routine treatment, bid; } \\
\text { hemoperfusion + routine } \\
\text { treatment, bid }\end{array}$ & 28 & 5,7 \\
\hline Liu JX & 2018 & 56 & $31 / 25$ & $18-69$ & $17-56$ & $\begin{array}{l}\text { Xuebijing + hemoperfusion } \\
\text { + routine treatment, bid; } \\
\text { hemoperfusion + routine } \\
\text { treatment, bid }\end{array}$ & 5 & 3,8 \\
\hline
\end{tabular}

Note: (I) ALT; (II) creatinine; (III) $\mathrm{PaO}_{2}$; (IV) $\mathrm{SpO}_{2}$; (V) 7-day survival rate; (VI) CRP; (VII) 14-day survival rate; (VIII) PaCO . ALT, alanine aminotransferase; $\mathrm{PaO}_{2}$, partial pressure; $\mathrm{SpO}_{2}$, oxygen saturation; CRP, C-reactive protein; $\mathrm{PaCO}_{2}$, partial pressure of carbon dioxide. 


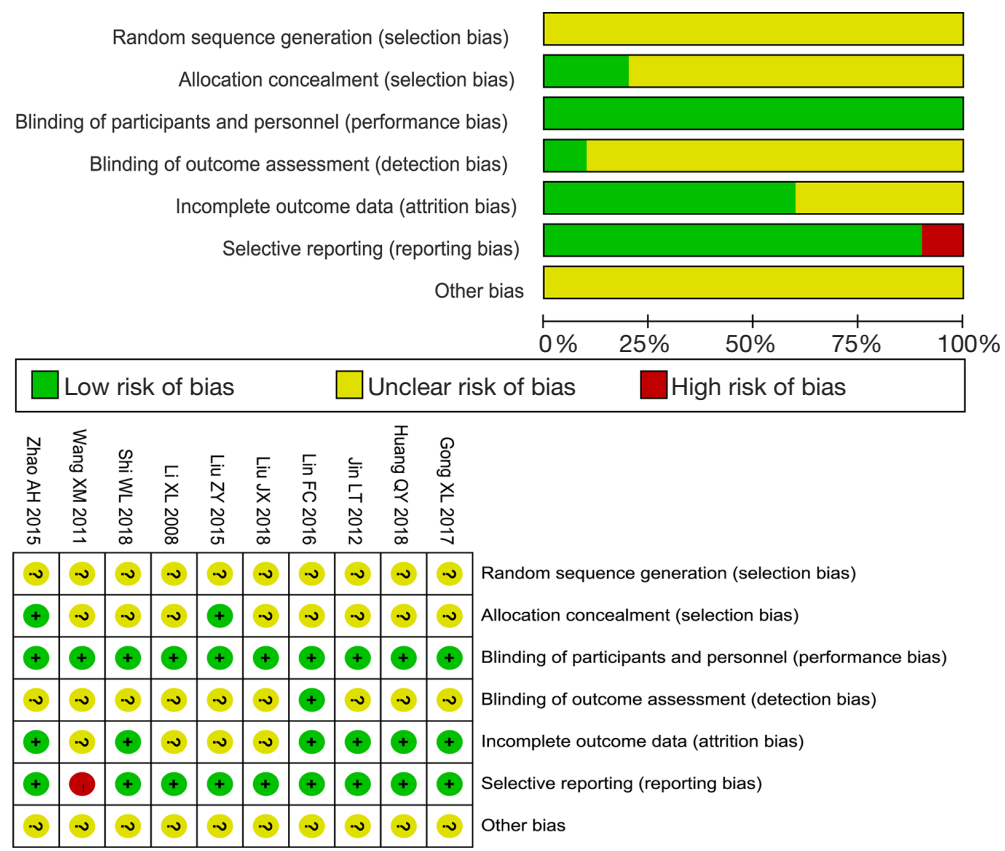

Figure 2 Methodological quality evaluation of included studies. (A) Risk of bias graph; (B) risk of bias summary.

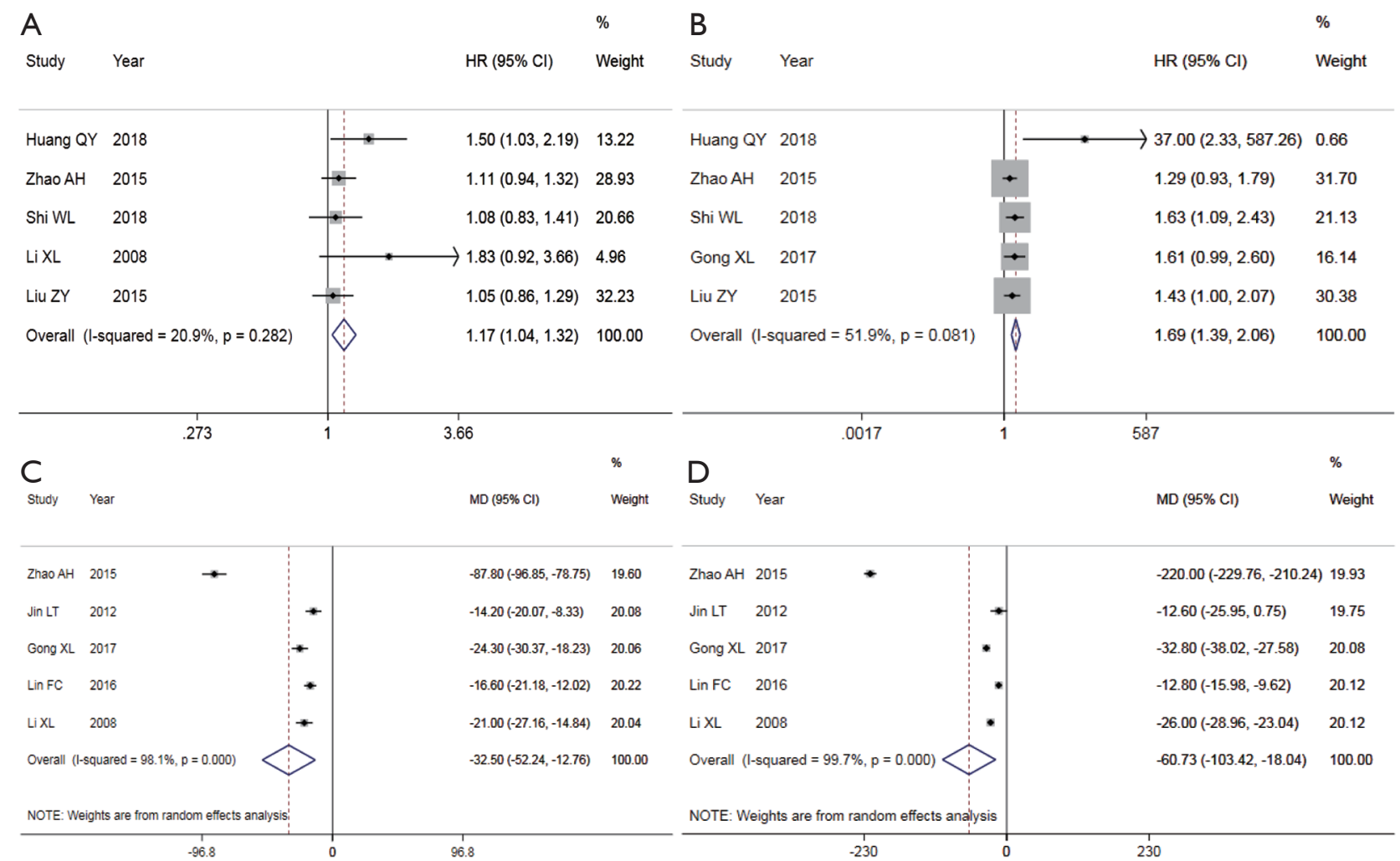

Figure 3 Forest plot of the effect of Xuebijing combined with plasma perfusion on 7-day survival rate, 14-day survival rate, ALT, and creatinine. (A) A forest plot of 7-day survival rate; (B) a forest plot of 14-day survival rate; (C) a forest plot of ALT; (D) a forest plot of creatinine. ALT, alanine aminotransferase. 


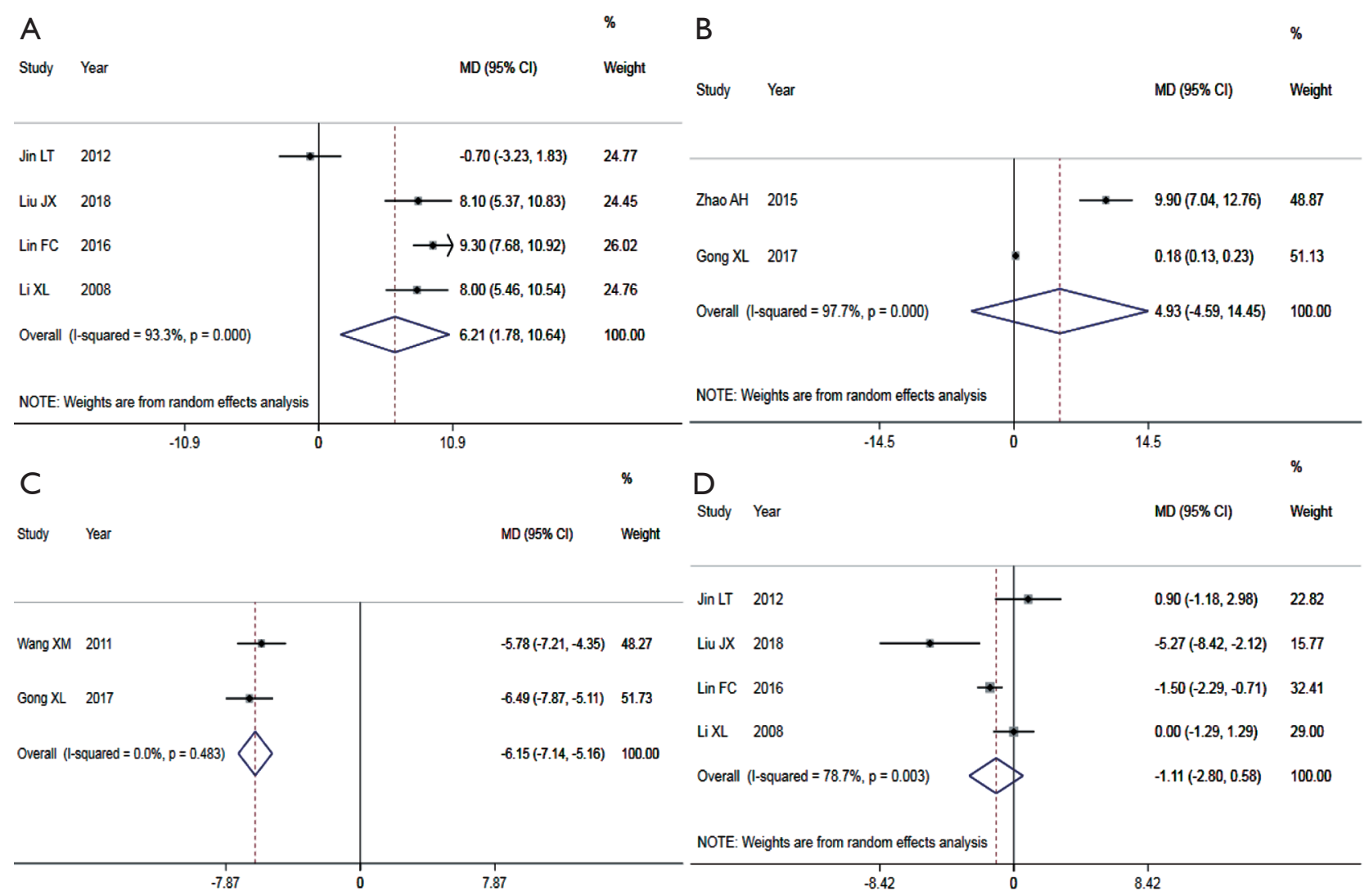

Figure 4 Forest plot of the effect of Xuebijing combined with plasma perfusion on $\mathrm{PaO}_{2}, \mathrm{SpO}_{2}, \mathrm{CRP}$, and $\mathrm{PaCO}$. (A) A forest plot of PaO ; (B) a forest plot of $\mathrm{SpO}_{2}$; (C) a forest plot of CRP; (D) a forest plot of $\mathrm{PaCO}_{2}$. $\mathrm{PaO}_{2}$, partial pressure; SpO , oxygen saturation; CRP, C-reactive protein; $\mathrm{PaCO}_{2}$, partial pressure of carbon dioxide.

indexes in this study were relatively robust.

\section{Publication bias analysis}

Stata15.0 software was used for Egger's test for each outcome index. If $\mathrm{P}>0.05$, there was considered to be no publication bias in the study (Table 2).

\section{Discussion}

Xuebijing combined with hemoperfusion has been widely used in clinical practice, and has been shown to effectively improve the important organ function of patients and reduce the incidence of complications $(8,23)$. Xuebijing contains traditional Chinese medicine components that have the effects of promoting blood circulation, removing blood stasis, clearing away heat and toxic materials, and treating bacteria and toxins simultaneously (16). Xuebijing directly delivers drugs to blood circulation through intravenous drip, and achieves therapeutic effect mainly by regulating inflammatory reaction and antioxidant stress (14). Among its ingredients, danshen root, chuanxiong rhizome, safflower, and angelica induce anti-inflammatory effects (5), inhibit the release of pro-inflammatory factors such as TNF- $\alpha$, IL-1, IL-6, etc., promote the expression of antiinflammatory factors (such as IL-10), rebuild the balance of anti-inflammatory transmitters of inflammatory transmitters, reduce inflammatory reactions, and protect viscera (34). Xuebijing can also reduce the occurrence of excessive oxidation, has a strong scavenging effect on oxygen-free radicals, and can significantly increase the activity of SOD $(8,13)$. By reducing the content of MDA, a von Willebrand factor (vWF), endothelin (ET) in plasma and by increasing the activity of SOD, Xuebijing can eliminate free radicals, reduce oxidative damage caused by excessive free radical release, protect vascular endothelium, 
Table 2 Main results of the meta-analysis

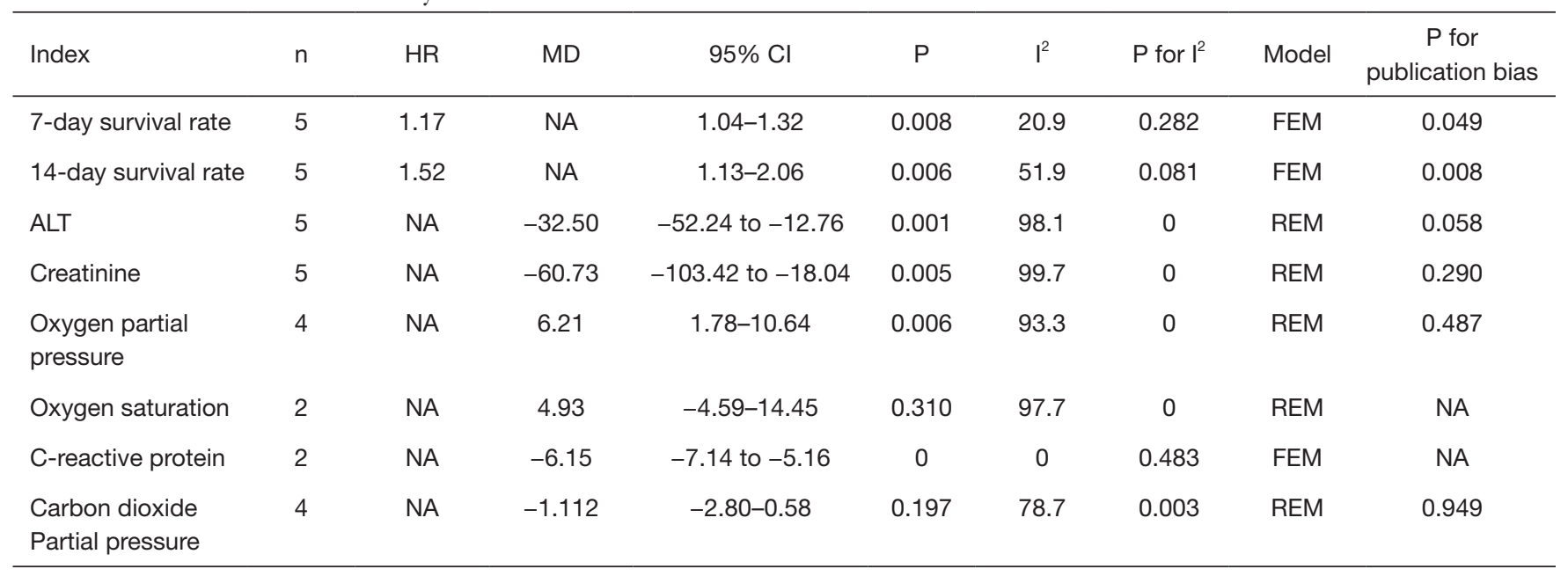

$\mathrm{HR}$, hazard ratio; MD, mean difference; $\mathrm{Cl}$, confidence interval; $\mathrm{ALT}$, alanine aminotransferase.

reduce vascular permeability, inhibit extracellular matrix deposition, and reduce lung fibrosis damage (22). Hemoperfusion can effectively remove inflammatory factors and other toxic substances in the blood circulation, reduce the concentration of PQ in the body, and synergize with Xuebijing to ameliorate damage to the liver, kidney, and lung, thereby bringing down the mortality rate of poisoned patients $(14,23)$.

There were 10 studies included in this article (23,25-33) comprising 636 patients. Meta-analysis results showed that Xuebijing combined with hemoperfusion can reduce ALT and creatinine levels, improve liver and kidney function, increase $\mathrm{PaO}_{2}$, and enhance oxygenation level to a certain extent. The results showed that Xuebijing combined with hemoperfusion decreased CRP, regulated inflammatory reaction, and further improved 7 - and 14-day survival rates of patients; this is consistent with the relevant research results of recent years $(8,14,23)$. Therefore, this meta-analysis provides further evidence that Xuebijing combined with hemoperfusion is an effective treatment for PQ. From the analysis results, Xuebijing combined with hemoperfusion can bring greater benefits to PQ patients. However, the results of ALT, creatinine, $\mathrm{PaO}_{2}, \mathrm{SpO}_{2}$, and $\mathrm{PaCO}_{2}$ were heterogeneous in the study. The possible sources were differences in sample size, laboratory testing methods, and instruments. We suggest that subsequent studies strictly include or exclude certain criteria, and that some factors causing clinical heterogeneity should be excluded as much as possible, such as differences in sample size, laboratory testing methods, and instruments.
Although this study confirmed the efficacy of Xuebijing combined with hemoperfusion in the treatment of PQ, meta-analysis is based on published studies, so the conclusion is greatly influenced by the quality of the included literature. In the studies included in this article, the following issues were present: (I) the study only mentioned "random allocation", and the method of generating random allocation was not reported in detail. (II) There was a substantial difference in the sample size of some included studies, and only Chinese literature was included, which might have introduced language bias. (III) All the included studies were completed in China, and there was a lack of efficacy evaluation for patients of different countries and races, and thus the results cannot be widely popularized. (IV) The heterogeneity of some outcome indexes was relatively high, which might have been due to differences in patients' age, gender, laboratory testing instruments, methods, etc. (V) Conventional treatment strategies might have been inconsistent across different studies, which could have had an influence on the results.

In conclusion, although this study has certain limitations, it conclusively found that Xuebijing combined with hemoperfusion does have certain therapeutic effects on PQ. However, the above conclusions still need more highquality clinical controlled trials for verification. Further research needs to include RCTs with reasonably designed large samples from multiple centers and a long followup time to verify the efficacy and safety. This way, a solid theoretical foundation for the clinical application of Xuebijing combined with hemoperfusion can be finally laid. 


\section{Acknowledgments}

Thanks to Guangzhou Yujia Biotechnology Co., Ltd for technical support.

Funding: Funding was received from the Foshan Science and Technology Innovation Project (2016AB002621) and the Science and Technology Program of Guangdong Province (2013B0218000038).

\section{Footnote}

Reporting Checklist: The authors have completed the PRISMA reporting checklist. Available at http://dx.doi. org/10.21037/apm-20-1247

Conflicts of Interest: All authors have completed the ICMJE uniform disclosure form (available at http://dx.doi. org/10.21037/apm-20-1247). The authors have no conflicts of interest to declare.

Ethical Statement: The authors are accountable for all aspects of the work in ensuring that questions related to the accuracy or integrity of any part of the work are appropriately investigated and resolved.

Open Access Statement: This is an Open Access article distributed in accordance with the Creative Commons Attribution-NonCommercial-NoDerivs 4.0 International License (CC BY-NC-ND 4.0), which permits the noncommercial replication and distribution of the article with the strict proviso that no changes or edits are made and the original work is properly cited (including links to both the formal publication through the relevant DOI and the license). See: https://creativecommons.org/licenses/by-nc-nd/4.0/.

\section{References}

1. Tong F, Tian Y, Shi H, et al. Study on the expression of nuclear factor-kappa $\mathrm{B}$ and inducible nitric oxide synthase in lung tissue of acute paraquat poisoned rats. Chinese Journal of Critical Care Medicine 2006;26:520-2.

2. Li X, Gao J, Wang A. Effects of Xuebijing Injection on Acute Severe Parquat Poisoning. China Pharmacy 2008;(32):57-9.

3. Nasr Isfahani S, Farajzadegan Z, Sabzghabaee AM, et al. Does hemoperfusion in combination with other treatments reduce the mortality of patients with paraquat poisoning more than hemoperfusion alone: A systematic review with meta-analysis. J Res Med Sci 2019;24:2.

4. Sabzghabaee AM, Eizadi-Mood N, Montazeri K, et al. Fatality in paraquat poisoning. Singapore Med J 2010;51:496-500.

5. Gong P, Lu Z, Xing J, et al. Traditional chinese medicine Xuebijing treatment is associated with decreased mortality risk of patients with moderate paraquat poisoning. PLoS One 2015;10:e0123504.

6. Gawarammana IB, Buckley NA. Medical management of paraquat ingestion. Br J Clin Pharmacol 2011;72:745-57.

7. Jones GM, Vale JA. Mechanisms of toxicity, clinical features, and management of diquat poisoning: a review. J Toxicol Clin Toxicol 2000;38:123-8.

8. Liu Y, Li P. Effect of Xuebijing Injection Combined with Hemoperfusion on Paraquat Poisoning and Its Influence on CRP, MDA Levels of Serum and SOD Activity of Patients. Strait Pharmaceutical Journal 2019;31:130-1.

9. Chen J, Chen J, Tang X, et al. Clinical and Animal Experimental Observation on Compound Danshen, Scopolamine and Dexamethasone in Treating Paraquat Poisoning. Chinese Critical Care Medicine 1997;9:516-74.

10. Zhang Z. Changes of serum cytokine caused by acute paraquat poisoning and medical treatment study. Shandong University, 2007.

11. Hsieh YW, Lin JL, Lee SY, et al. Paraquat poisoning in pediatric patients. Pediatr Emerg Care 2013;29:487-91.

12. Cho JH, Yang DK, Kim L, et al. Inhaled nitric oxide improves the survival of the paraquat-injured rats. Vascul Pharmacol 2005;42:171-8.

13. Xu Dahai, Gao Jinying, Zhang Nan, et al. Progress in Comprehensive Treatment of Paraquat Poisoning. Chongqing Medicine 2011;40:2278-80.

14. Wu Y, Yu Y, Wang J. Protective effect of Xuebijing injection combined with hemoperfusion on organ injury in patients with severe paraquat poisoning. Hebei Medicine 2017;(11):129-32.

15. Deng J, Huo D, Wu Q, et al. Xuebijing for paraquat poisoning. Cochrane Database Syst Rev 2013;(7):CD010109.

16. Lu YQ, Gu LH, Huang WD. Effect of Xuebijing injection on peripheral T-lymphocyte subpopulations in patients with severe trauma. Chinese Journal of Traumatology (English Edition) 2010;13:72-6.

17. Huang H, Ji L, Song S, et al. Identification of the major constituents in Xuebijing injection by HPLC-ESI-MS. Phytochem Anal 2011;22:330-8.

18. Zhang Y, Hu W, Ren S, et al. Simultaneous Determination of Multicomponent in Xuebijing by HPLC. Chinese 
Journal of Spectroscopy Laboratory 2013;30:271-4.

19. Lee JY, Chang EJ, Kim HJ, et al. Antioxidative flavonoids from leaves of Carthamus tinctorius. Arch Pharm Res 2002;25:313-9.

20. Lee SC, Kwon YS, Son KH, et al. Antioxidative constituents from Paeonia lactiflora. Arch Pharm Res 2005;28:775-83.

21. Kexiu Q. The protective role of Xuebijing on patients with acute paraquat poisoning. Acta Universitatis Medicinalis Anhui, 2011.

22. Liu Y. Research Progress on Mechanism of Xuebijing Injection Against Paraquat Poisoning. Tianjin Journal of Traditional Chinese Medicine 2013;30:72-4.

23. Lin F, Wang R, Li S, et al. Exploring Effectiveness of Xuebijing Injection Combined with Hemoperfusion on Acute Paraquat Poisoning. Chinese Archives of Traditional Medicine 2016;34:1714-6.

24. Julian PT, Higgin S, Sally G. Cochrane Handbook for Systematic Reviews of Interventions (EB/OL). The Cochrane Collaboration, 2013.

25. Li X, Gao J, Wang A. Effects of Xuebijing injection combined with hemoperfusion on the acute paraquat poisoning at the early stage. Chinese Journal of Critical Care Medicine 2008;28:78-80.

26. Wang X, Shi Y, Dong Y, et al. Clinical effect of acute paraquat poisoning treatment with xuebijing injection combined with hemoperfusion. Sichuan Medicine 2011;32:817-9.

27. Jin L, Zhu J, Huang J. Xuebijing Combined Hemoperfusion in Treating 35 Cases of Acute Paraquat

Cite this article as: Fu Y, Yan M, Zeng X, Xie C, Xu W, Feng J, Jiang J. Meta-analysis of the efficacy of Xuebijing combined with hemoperfusion in treating paraquat poisoning. Ann Palliat Med 2020;9(4):2152-2162. doi: 10.21037/apm-20-1247
Poisoning. China Pharmaceuticals 2012;21:84-5.

28. Zhao A, Guan Q, Chen A, et al. Clinical study of Xuebijing injection combined with hemoperfusion on the treatment of acute paraquat poisoning under different APACHEII score. Journal of Clinical Emergency 2015;16:771-3.

29. Liu Z. Xuebijing combined hemoperfusion efficacy in patients with early application of paraquat poisoning pulmonary fibrosis. Anhui Medical University, 2015.

30. Gong Xiaoliang, Fan Feifei, Gao Xinxing, et al. Effect Observation of Xuebijing Injection Combined with Hemoperfusion in Early Treatment of Acute Paraquat Poisoning. Medical \& Pharmaceutical Journal of Chinese People's Liberation Army 2017;29:14-7.

31. Huang Q. Experience in Treatment of 60 cases of Paraquat Intoxication. Chinese and Foreign Medical Research 2018;16:133-4.

32. Shi W. Prevention effect analysis of Xuebiqing combined with blood perfusion on chronic lung injury in 68 cases with acute PQ poisoning. Journal of Community Medicine 2018;16:27-9.

33. Liu J, Zheng B. Therapeutic effect of early combined application of Xuebijing and hemoperfusion in pulmonary fibrosis of patients with paraquat poisoning. Clinical Research and Practice 2018;3:32-4.

34. Liu Z, Dong W, Yu Z, et al. Efficacy of Xuebijing Injection in Treatment of Acute Paraquat Poisoning:A Metaanalysis. Herald of Medicine 2018;37:1267-75.

(English Language Editor: J. Gray) 